\title{
A Formula for the Energy of Circulant Graphs with Two Generators
}

\author{
Justine Louis \\ Section de Mathématiques, Université de Genève, 1211 Geneva, Switzerland \\ Correspondence should be addressed to Justine Louis; justine.louis@unige.ch \\ Received 6 June 2016; Accepted 26 July 2016 \\ Academic Editor: Hong J. Lai
}

Copyright (C) 2016 Justine Louis. This is an open access article distributed under the Creative Commons Attribution License, which permits unrestricted use, distribution, and reproduction in any medium, provided the original work is properly cited.

We derive closed formulas for the energy of circulant graphs generated by 1 and $\gamma$, where $\gamma \geqslant 2$ is an integer. We also find a formula for the energy of the complete graph without a Hamilton cycle.

Let $1 \leqslant \gamma_{1} \leqslant \cdots \leqslant \gamma_{d}$ be integers. The circulant graph $C_{n}^{\gamma_{1}, \ldots, \gamma_{d}}$ generated by $\gamma_{1}, \ldots, \gamma_{d}$ on $n$ vertices labelled $0,1, \ldots, n-1$ is the 2D-regular graph such that, for all $v \in \mathbb{Z} / n \mathbb{Z}, v$ is connected to $v+\gamma_{i} \bmod n$ and to $v-\gamma_{i} \bmod n$, for all $i=$ $1, \ldots, d$. The adjacency matrix $A=\left(A_{i j}\right)$ of a graph on $n$ vertices is the $n \times n$ matrix with rows and columns indexed by the vertices such that $A_{i j}$ is the number of edges connecting vertices $i$ and $j$. Let $\lambda_{k}, k=1, \ldots, n$, denote the eigenvalues of the adjacency matrix. The energy of a graph $G$ on $n$ vertices is defined by the sum of the absolute values of the eigenvalues of $A$; that is,

$$
E(G)=\sum_{k=1}^{n}\left|\lambda_{k}\right|
$$

The energy of circulant graphs and integral circulant graphs is widely studied; see, for example, [1-4]. It has interesting applications in theoretical chemistry; namely, it is related to the $\pi$-electron energy of a conjugated carbon molecule; see [5]. In the following theorem, we give a formula for the energy of circulant graphs with two generators, 1 and $\gamma, \gamma \geqslant 2$. The formula is interesting as $n$ is larger than $\gamma$.

Theorem 1. Let $D_{n}(x)$ denote the Dirichlet kernel. The energy of the circulant graph $C_{n}^{1,2}$ is given by

$$
E\left(C_{n}^{1,2}\right)=4\left(D_{\lfloor n / 6\rfloor}\left(\frac{2 \pi}{n}\right)+D_{\lfloor n / 6\rfloor}\left(\frac{4 \pi}{n}\right)\right)
$$

For $\gamma \geqslant 3$, the energy of the circulant graph $C_{n}^{1, \gamma}$ is given by

$$
\begin{aligned}
& E\left(C_{n}^{1, \gamma}\right)=4 \sum_{m \in\{1, \gamma\}}\left(\sum_{l=0}^{\lceil\gamma / 2\rceil-1} D_{\lfloor(2 l+1) n /(2(\gamma+1))\rfloor}\left(\frac{2 \pi m}{n}\right)\right. \\
& \left.-\sum_{l=0}^{\lceil\gamma / 2\rceil-2} D_{\lfloor(2 l+1) n /(2(\gamma-1))\rfloor}\left(\frac{2 \pi m}{n}\right)\right),
\end{aligned}
$$

where $\lfloor x\rfloor$ denotes the greatest integer smaller than or equal to $x$ and $\lceil x\rceil$ denotes the smallest integer greater than or equal to $x$.

Proof. The adjacency matrix of a circulant graph is circulant; it follows that the eigenvalues of $C_{n}^{1, \gamma}$ are given by $\lambda_{k}=$ $2 \cos (2 \pi k / n)+2 \cos (2 \pi \gamma k / n), k=0, \ldots, n-1$ (see [6]). The energy of $C_{n}^{1, \gamma}$ is then given by

$$
E\left(C_{n}^{1, \gamma}\right)=2 \sum_{k=0}^{n-1}\left|\cos \left(\frac{2 \pi k}{n}\right)+\cos \left(\frac{2 \pi \gamma k}{n}\right)\right| .
$$

Let $\gamma=2$. The two roots of the equation $\cos x+\cos (2 x)=0$ for $x \in[0, \pi]$ are $\pi / 3$ and $\pi$. We write the energy as

$$
E\left(C_{n}^{1,2}\right)=4+4 \sum_{k=1}^{\lceil n / 2\rceil-1}\left|\cos \left(\frac{2 \pi k}{n}\right)+\cos \left(\frac{4 \pi k}{n}\right)\right|
$$




$$
\begin{aligned}
= & 4+4 \sum_{k=1}^{\lfloor n / 6\rfloor}\left(\cos \left(\frac{2 \pi k}{n}\right)+\cos \left(\frac{4 \pi k}{n}\right)\right) \\
& -4 \sum_{k=\lfloor n / 6\rfloor+1}^{\lceil n / 2\rceil-1}\left(\cos \left(\frac{2 \pi k}{n}\right)+\cos \left(\frac{4 \pi k}{n}\right)\right) .
\end{aligned}
$$

The sum of $\cos (k x)$ over consecutive $k$ 's can be expressed in terms of the Dirichlet kernel; namely,

$$
D_{n}(x)=1+2 \sum_{k=1}^{n} \cos (k x)=\frac{\sin ((n+1 / 2) x)}{\sin (x / 2)}
$$

As a consequence,

$$
2 \sum_{k=n+1}^{m} \cos (k x)=D_{m}(x)-D_{n}(x) .
$$

The energy of $C_{n}^{1,2}$ is thus given by

$$
\begin{aligned}
E\left(C_{n}^{1,2}\right)= & 4 D_{\lfloor n / 6\rfloor}\left(\frac{2 \pi}{n}\right)+4 D_{\lfloor n / 6\rfloor}\left(\frac{4 \pi}{n}\right) \\
& -2 D_{\lceil n / 2\rceil-1}\left(\frac{2 \pi}{n}\right)-2 D_{\lceil n / 2\rceil-1}\left(\frac{4 \pi}{n}\right) .
\end{aligned}
$$

The formula then follows from the fact that, for odd $n$, $D_{(n-1) / 2}(2 \pi m / n)=0$ for $m=1,2$, and, for even $n, D_{n / 2-1}(2 \pi /$ $n)=1$ and $D_{n / 2-1}(4 \pi / n)=-1$.
Let $\gamma \geqslant 3$. For odd $\gamma$, the $\gamma$ solutions of the equation $\cos x+$ $\cos \gamma x=0$ for $x \in[0, \pi]$ are given in the increasing order by $\pi /(\gamma+1), \pi /(\gamma-1), 3 \pi /(\gamma+1), 3 \pi /(\gamma-1), \ldots,(\gamma-2) \pi /(\gamma-$ $1), \gamma \pi /(\gamma+1)$. For even $\gamma$, they are given by $\pi /(\gamma+1), \pi /(\gamma-$ $1), 3 \pi /(\gamma+1), 3 \pi /(\gamma-1), \ldots,(\gamma-3) \pi /(\gamma-1),(\gamma-1) \pi /(\gamma+1), \pi$. Let $n$ be odd. We split the sum over $k$ of cosines to group the positive terms together and the negative terms together. The energy is given by

$$
\begin{aligned}
& E\left(C_{n}^{1, \gamma}\right)=4+4 \sum_{k=1}^{(n-1) / 2}\left|\cos \left(\frac{2 \pi k}{n}\right)+\cos \left(\frac{2 \pi \gamma k}{n}\right)\right| \\
& =4+4 \sum_{k=1}^{\lfloor n /(2(\gamma+1)\rfloor\rfloor}\left(\cos \left(\frac{2 \pi k}{n}\right)+\cos \left(\frac{2 \pi \gamma k}{n}\right)\right) \\
& +4 \sum_{l=0}^{\lceil\gamma / 2\rceil-2} \sum_{k=\lfloor(2 l+1) n /(2(\gamma-1))\rfloor+1}^{\lfloor(2 l+3) n /(2(\gamma+1))\rfloor}\left(\cos \left(\frac{2 \pi k}{n}\right)\right. \\
& \left.+\cos \left(\frac{2 \pi \gamma k}{n}\right)\right) \sum_{l \gamma / 2\rceil-1}^{\lfloor(2 l+1) n /(2(\gamma-1))\rfloor}\left(\cos \left(\frac{2 \pi k}{n}\right)\right. \\
& -4 \sum_{l=0}^{k=\lfloor(2 l+1) n /(2(\gamma+1))\rfloor+1} \\
& \left.+\cos \left(\frac{2 \pi \gamma k}{n}\right)\right) .
\end{aligned}
$$

Writing the above relation in terms of Dirichlet kernels, we have

$$
\begin{aligned}
E & \left(C_{n}^{1, \gamma}\right)=2 \sum_{m \in\{1, \gamma\}}\left(D_{\lfloor n /(2(\gamma+1))\rfloor}\left(\frac{2 \pi m}{n}\right)+\sum_{l=0}^{\lceil\gamma / 2\rceil-2}\left(D_{\lfloor(2 l+3) n /(2(\gamma+1))\rfloor}\left(\frac{2 \pi m}{n}\right)-D_{\lfloor(2 l+1) n /(2(\gamma-1))\rfloor}\left(\frac{2 \pi m}{n}\right)\right)\right. \\
& \left.-\sum_{l=0}^{\lceil\gamma / 2\rceil-1}\left(D_{\lfloor(2 l+1) n /(2(\gamma-1))\rfloor}\left(\frac{2 \pi m}{n}\right)-D_{\lfloor(2 l+1) n /(2(\gamma+1))\rfloor}\left(\frac{2 \pi m}{n}\right)\right)\right) .
\end{aligned}
$$

Hence,

$$
\begin{aligned}
& E\left(C_{n}^{1, \gamma}\right)=\sum_{m \in\{1, \gamma\}}\left(4 \sum_{l=0}^{\lceil\gamma / 2\rceil-1} D_{\lfloor(2 l+1) n /(2(\gamma+1))\rfloor}\left(\frac{2 \pi m}{n}\right)\right. \\
& -4 \sum_{l=0}^{\lceil\gamma / 2\rceil-2} D_{\lfloor(2 l+1) n /(2(\gamma-1))\rfloor}\left(\frac{2 \pi m}{n}\right) \\
& \left.-2 D_{\lfloor n / 2\rfloor}\left(\frac{2 \pi m}{n}\right)\right) .
\end{aligned}
$$

The formula follows from the fact that $D_{\lfloor n / 2\rfloor}(2 \pi m / n)=0$ for $m=1, \gamma$.
Let $n$ be even. As for the case when $n$ is odd, we write the energy as follows:

$$
\begin{aligned}
E\left(C_{n}^{1, \gamma}\right)= & 4\left(1+\delta_{\gamma \text { odd }}\right) \\
& +4 \sum_{k=1}^{n / 2-1}\left|\cos \left(\frac{2 \pi k}{n}\right)+\cos \left(\frac{2 \pi \gamma k}{n}\right)\right|,
\end{aligned}
$$

where $\delta_{\gamma \text { odd }}=1$ if $\gamma$ is odd and 0 otherwise.

For even $\gamma$, relations (9), (10), and (11) also hold. The theorem then follows from the fact that $D_{n / 2}(2 \pi / n)=-1$ and $D_{n / 2}(2 \pi \gamma / n)=1$. For odd $\gamma$, we have

$$
\begin{aligned}
& E\left(C_{n}^{1, \gamma}\right)=8+4 \sum_{k=1}^{\lfloor n /(2(\gamma+1))\rfloor}\left(\cos \left(\frac{2 \pi k}{n}\right)\right. \\
& \left.+\cos \left(\frac{2 \pi \gamma k}{n}\right)\right)
\end{aligned}
$$




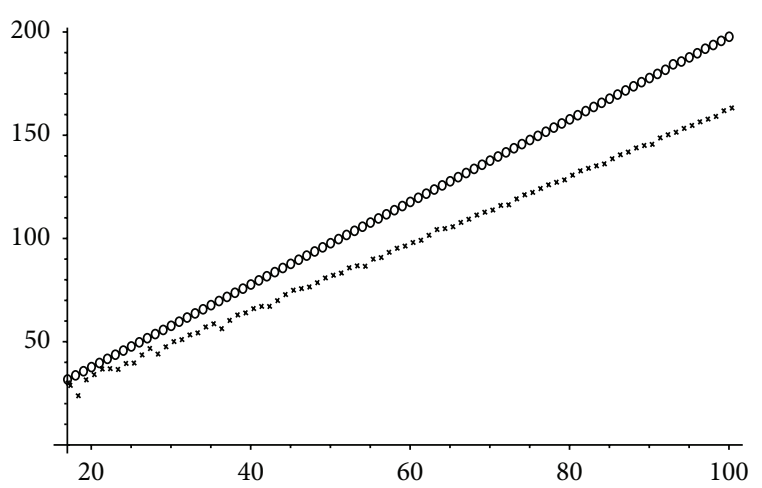

(a) Energy of $C_{n}^{1,8}$ (crosses) and of $K_{n}$ (circles) with respect to $n$

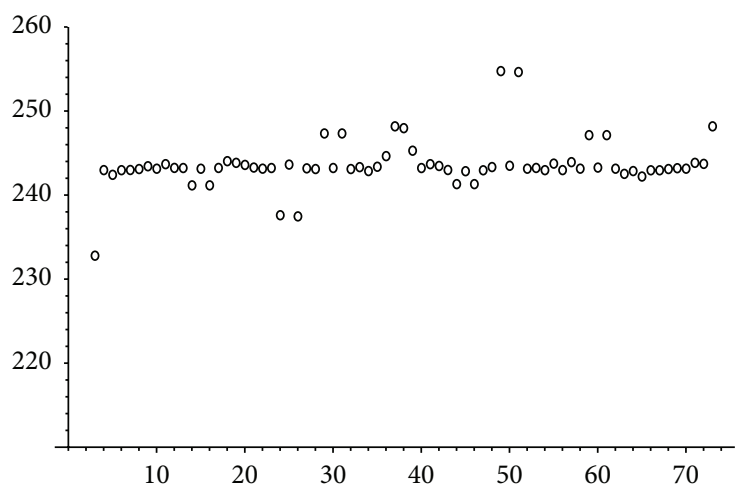

(b) Energy of $C_{150}^{1, \gamma}$ with respect to $\gamma$

FIGURE 1: Energy of circulant graphs.

$$
\begin{aligned}
& +4 \sum_{l=0}^{\lceil\gamma / 2\rceil-2} \sum_{k=\lfloor(2 l+1) n /(2(\gamma-1))\rfloor+1}^{\lfloor(2 l+3) n /(2(\gamma+1))\rfloor}\left(\cos \left(\frac{2 \pi k}{n}\right)\right. \\
& \left.+\cos \left(\frac{2 \pi \gamma k}{n}\right)\right) \\
& -4 \sum_{l=0}^{\lceil\gamma / 2\rceil-2} \sum_{k=\lfloor(2 l+1) n /(2(\gamma+1))\rfloor+1}^{\lfloor(2 l+1) n /(2(\gamma-1))\rfloor}\left(\cos \left(\frac{2 \pi k}{n}\right)\right.
\end{aligned}
$$

$$
\begin{aligned}
& \left.+\cos \left(\frac{2 \pi \gamma k}{n}\right)\right) \\
& -4 \sum_{k=\lfloor(2\lceil\gamma / 2\rceil-1) n /(2(\gamma+1))\rfloor+1}^{n / 2-1}\left(\cos \left(\frac{2 \pi k}{n}\right)\right. \\
& \left.+\cos \left(\frac{2 \pi \gamma k}{n}\right)\right) .
\end{aligned}
$$

Expressing it in terms of Dirichlet kernels, we have

$$
\begin{aligned}
& E\left(C_{n}^{1, \gamma}\right)=4+2 \sum_{m \in\{1, \gamma\}}\left(D_{\lfloor n /(2(\gamma+1))\rfloor}\left(\frac{2 \pi m}{n}\right)+\sum_{l=0}^{\lceil\gamma / 2\rceil-2}\left(D_{\lfloor(2 l+3) n /(2(\gamma+1))\rfloor}\left(\frac{2 \pi m}{n}\right)-D_{\lfloor(2 l+1) n /(2(\gamma-1))\rfloor}\left(\frac{2 \pi m}{n}\right)\right)\right. \\
& \left.\quad-\sum_{l=0}^{\lceil\gamma / 2\rceil-2}\left(D_{\lfloor(2 l+1) n /(2(\gamma-1))\rfloor}\left(\frac{2 \pi m}{n}\right)-D_{\lfloor(2 l+1) n /(2(\gamma+1))\rfloor}\left(\frac{2 \pi m}{n}\right)\right)-D_{n / 2-1}\left(\frac{2 \pi m}{n}\right)+D_{\lfloor(2\lceil\gamma / 2\rceil-1) n /(2(\gamma+1))\rfloor}\left(\frac{2 \pi m}{n}\right)\right) .
\end{aligned}
$$

The theorem follows from the fact that $D_{n / 2-1}(2 \pi m / n)=1$ for $m=1, \gamma$.

A graph is called hyperenergetic if its energy is greater than the one of the complete graph $K_{n}$. The eigenvalues of the adjacency matrix of $K_{n}$ are given by $n-1$ and -1 with multiplicity $n-1$, so that its energy is given by $E\left(K_{n}\right)=$ $2(n-1)$.

Figure 1(a) shows how the energy of $C_{n}^{1, \gamma}$ grows with respect to $n$ for $\gamma=8$. We see that it is not hyperenergetic and that the energy grows more or less linearly with respect to $n$. Figure 1(b) shows the energy of $C_{n}^{1, \gamma}$ with fixed $n$ as $\gamma$ varies. We observe that the energy stays more or less constant independently of $\gamma$.

As a consequence of the theorem, we can carry out the sum of the Dirichlet kernels when the number of vertices is proportional to $2(\gamma-1)(\gamma+1)$.

Corollary 2. Given integers $\gamma \geqslant 3$ and $\alpha \geqslant 1$, the energy of the circulant graph $C_{2 \alpha(\gamma-1)(\gamma+1)}^{1, \gamma}$ is given by

$$
\begin{gathered}
E\left(C_{2 \alpha(\gamma-1)(\gamma+1)}^{1, \gamma}\right)=4 \sum_{m \in\{1, \gamma\}}\left(\frac{\sin (\pi m(\lceil\gamma / 2\rceil+1 /(2 \alpha(\gamma-1))) /(\gamma+1)) \sin (\lceil\gamma / 2\rceil \pi m /(\gamma+1))}{\sin (\pi m /(2 \alpha(\gamma-1)(\gamma+1))) \sin (\pi m /(\gamma+1))}\right. \\
\left.-\frac{\sin (\pi m(\lceil\gamma / 2\rceil-1+1 /(2 \alpha(\gamma+1))) /(\gamma-1)) \sin ((\lceil\gamma / 2\rceil-1) \pi m /(\gamma-1))}{\sin (\pi m /(2 \alpha(\gamma-1)(\gamma+1))) \sin (\pi m /(\gamma-1))}\right) .
\end{gathered}
$$


Proof. Let $a \geqslant 1$ and $K \geqslant 0$ be integers. The sum over $k$ of Dirichlet kernels of index $(2 k+1) a$ is given by

$$
\sum_{k=0}^{K} D_{(2 k+1) a}(x)=\sum_{k=0}^{K} \frac{\sin (((2 k+1) a+1 / 2) x)}{\sin (x / 2)} .
$$

By multiplying the summation by $\sin (a x) / \sin (a x)$ and using the trigonometric identity $2 \sin \theta \sin \phi=\cos (\theta-\phi)-\cos (\theta+$ $\phi)$, we have

$$
\begin{aligned}
& \sum_{k=0}^{K} D_{(2 k+1) a}(x) \\
& \quad=\frac{\cos (x / 2)-\cos (((2 K+2) a+1 / 2) x)}{2 \sin (x / 2) \sin (a x)} \\
& \quad=\frac{\sin (((2 K+2) a+1) x / 2) \sin ((K+1) a x)}{\sin (x / 2) \sin (a x)} .
\end{aligned}
$$

The corollary then follows by applying the above relation first with $a=\alpha(\gamma-1), K=\lceil\gamma / 2\rceil-1$ and second with $a=\alpha(\gamma+$ $1), K=\lceil\gamma / 2\rceil-2$, and $x=2 \pi m / n, m \in\{1, \gamma\}$.

In [7], the author considered the graphs $K_{n}-H$, where $K_{n}$ is the complete graph on $n$ vertices and $H$ is a Hamilton cycle of $K_{n}$, and asked whether these graphs are hyperenergetic. In [4], the authors showed that the energy of $K_{n}-H$ is given by

$$
E\left(K_{n}-H\right)=n-3+\sum_{k=1}^{n-1}\left|1+2 \cos \left(\frac{2 \pi k}{n}\right)\right|
$$

and that as $n$ goes to infinity, it is hyperenergetic. In the following proposition, we give a formula for it for all $n \geqslant 3$.

Proposition 3. For all $n \geqslant 3$, the energy of $K_{n}-H$ is given by

$$
\begin{aligned}
& E\left(K_{n}-H\right)=2\left(n-3-\left(\left\lfloor\frac{2 n}{3}\right\rfloor-\left\lfloor\frac{n}{3}\right\rfloor\right)\right)+2 \\
& \cdot \frac{\sin ((\lfloor n / 3\rfloor+1 / 2) 2 \pi / n)-\sin ((\lfloor 2 n / 3\rfloor+1 / 2) 2 \pi / n)}{\sin (\pi / n)} .
\end{aligned}
$$

Proof. We have

$$
\begin{aligned}
\sum_{k=1}^{n-1} \mid & +2 \cos \left(\frac{2 \pi k}{n}\right) \mid \\
= & \sum_{k=1}^{\lfloor n / 3\rfloor}\left(1+2 \cos \left(\frac{2 \pi k}{n}\right)\right) \\
& -\sum_{k=\lfloor n / 3\rfloor+1}^{\lfloor 2 n / 3\rfloor}\left(1+2 \cos \left(\frac{2 \pi k}{n}\right)\right) \\
& +\sum_{k=\lfloor 2 n / 3\rfloor+1}^{n-1}\left(1+2 \cos \left(\frac{2 \pi k}{n}\right)\right) \\
= & n-2-2\left(\left\lfloor\frac{2 n}{3}\right\rfloor-\left\lfloor\frac{n}{3}\right\rfloor\right)+2 D_{\lfloor n / 3\rfloor}\left(\frac{2 \pi}{n}\right) \\
& -2 D_{\lfloor 2 n / 3\rfloor}\left(\frac{2 \pi}{n}\right)+D_{n-1}\left(\frac{2 \pi}{n}\right) .
\end{aligned}
$$

Since $D_{n-1}(2 \pi / n)=-1$, the proposition follows.
By elementary analysis, one can show that $E\left(K_{n}-H\right)-$ $2(n-1)$ is increasing in $n$. As a consequence, we find that $K_{n}-$ $H$ are hyperenergetic for all $n \geqslant 10$. This has been previously found in [4].

\section{Competing Interests}

The author declares that there are no competing interests regarding the publication of this paper.

\section{Acknowledgments}

The author acknowledges support from the Swiss NSF Grant no. 200021_132528/1.

\section{References}

[1] S. R. Blackburn and I. E. Shparlinski, "On the average energy of circulant graphs," Linear Algebra and Its Applications, vol. 428, no. 8-9, pp. 1956-1963, 2008.

[2] A. Ilić and M. Bašić, "New results on the energy of integral circulant graphs," Applied Mathematics and Computation, vol. 218, no. 7, pp. 3470-3482, 2011.

[3] I. Shparlinski, "On the energy of some circulant graphs," Linear Algebra and Its Applications, vol. 414, no. 1, pp. 378-382, 2006.

[4] D. Stevanović and I. Stanković, "Remarks on hyperenergetic circulant graphs," Linear Algebra and Its Applications, vol. 400, no. 1-3, pp. 345-348, 2005.

[5] R. Balakrishnan and K. Ranganathan, A Textbook of Graph Theory, Universitext, Springer, New York, NY, USA, 2nd edition, 2012.

[6] N. Biggs, Algebraic Graph Theory, Cambridge Mathematical Library, Cambridge University Press, Cambridge, UK, 2nd edition, 1993.

[7] R. Balakrishnan, “The energy of a graph," Linear Algebra and its Applications, vol. 387, pp. 287-295, 2004. 


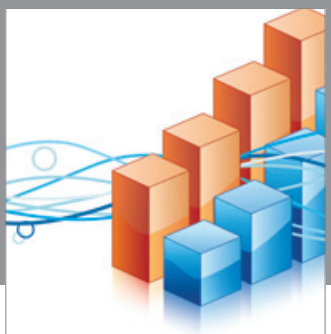

Advances in

Operations Research

vatem alat4

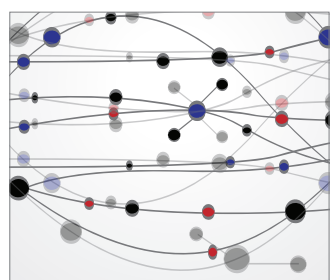

\section{The Scientific} World Journal
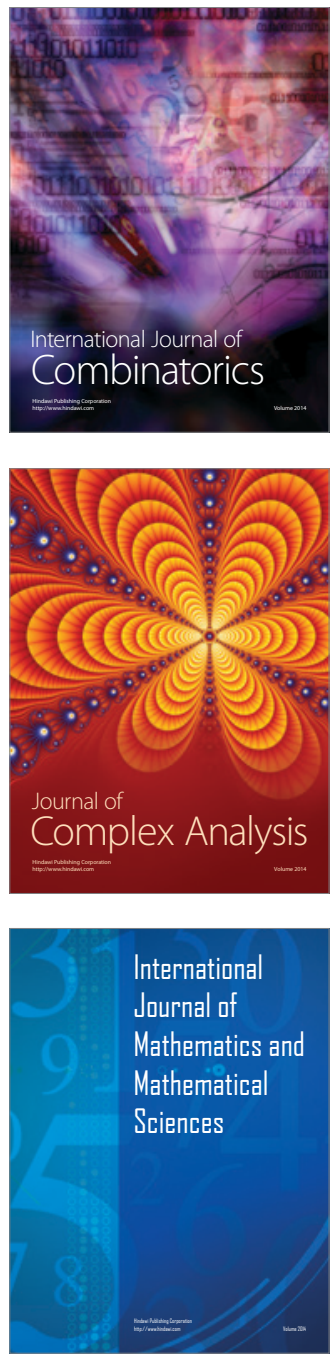
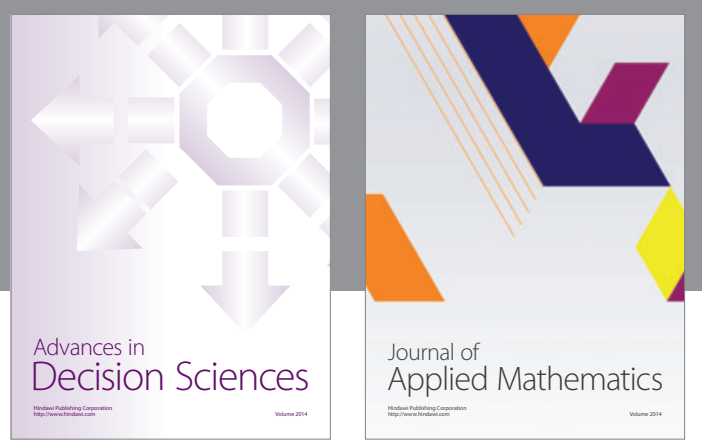

Algebra

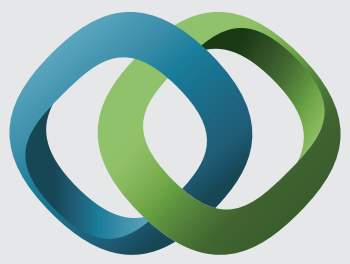

\section{Hindawi}

Submit your manuscripts at

http://www.hindawi.com
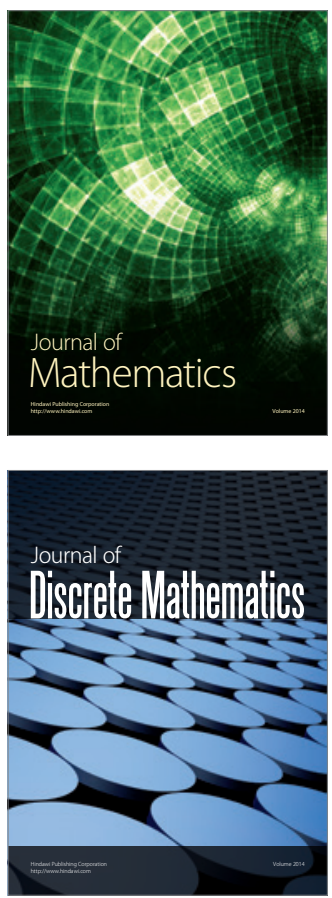

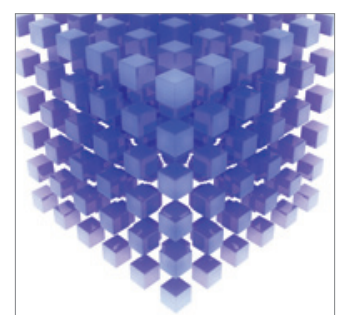

Mathematical Problems in Engineering
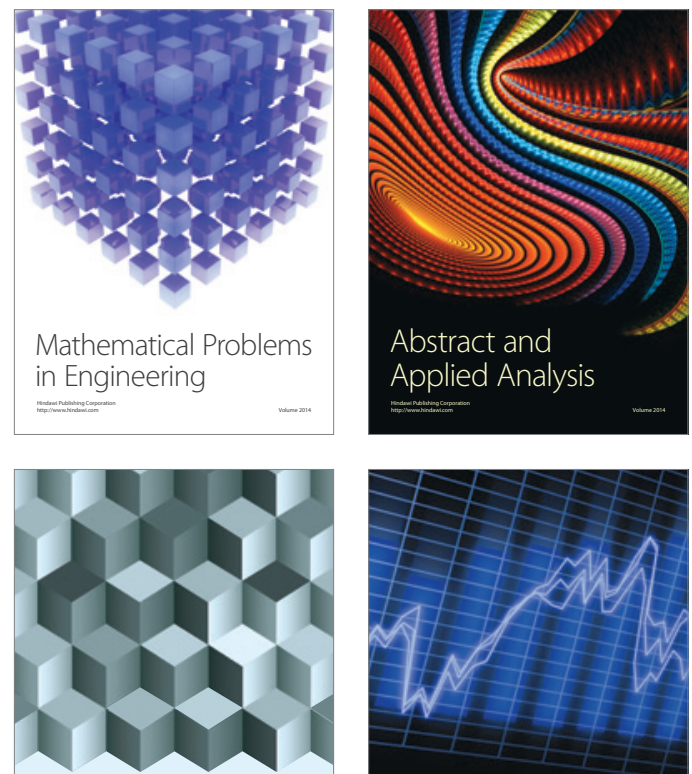

Journal of

Function Spaces

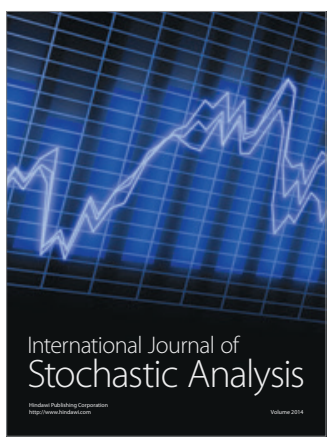

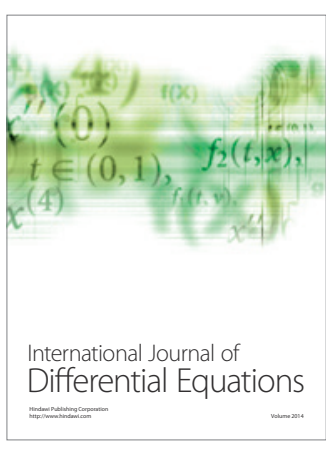
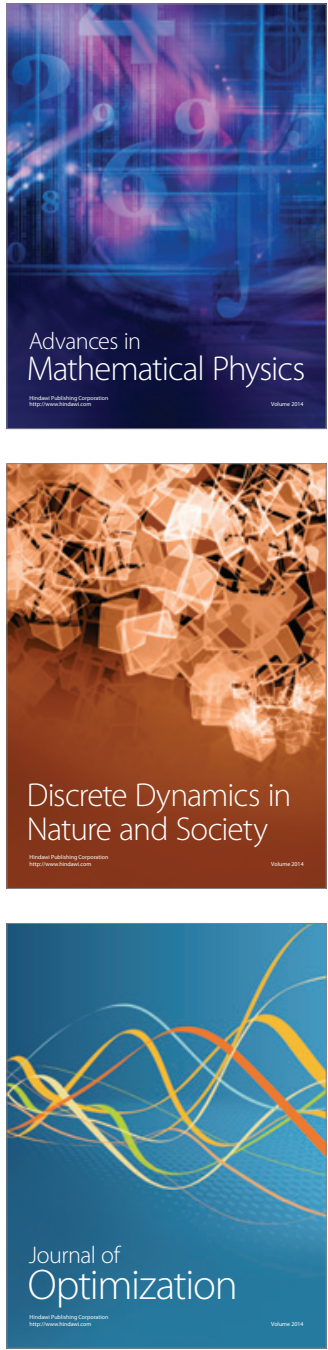